\title{
Corrigendum
}

\section{Differences in meal patterns and timing with regard to central obesity in the ANIBES ('Anthropometric data, macronutrients and micronutrients intake, practice of physical activity, socioeconomic data and lifestyles in Spain') Study - CORRIGENDUM}

\author{
Aránzazu Aparicio, Elena Rodríguez-Rodríguez, Javier Aranceta-Bartrina, Ángel Gil, \\ Marcela González-Gross, Lluis Serra-Majem, Gregorio Varela-Moreiras and \\ Rosa María Ortega
}

First published online 6 March 2018

First published online - 17 April 2017. DOI: https://doi.org/10.1017/S1368980017000635

We regret to announce this article included text from a Sofer et al. article (reference 8 in our article), that was not correctly cited in two of three instances. The ideas from Sofer et al. were only used in the introduction section, not in the rest of the investigation, and any omission of correct citation was not with any intent to plagiarise. The authors have re-written the relevant paragraphs and have cited them correctly below. The authors apologise for any inconvenience caused to the Journal or to the authors of the original article.

The second paragraph of the paper should read:

Changes in diet and physical activity are essential treatments in the strategies to reduce excess weight ${ }^{(5)}$; however, not all of these are equally effective ${ }^{(6)}$. In its 2013 guidelines, the Canadian Diabetes Association reviewed the efficacy of some of the more prominent dietary patterns or diets. The conclusion was that dietary patterns including vegetarian, the Mediterranean and the Dietary Approaches to Stop Hypertension diets could be recommended. In addition, certain popular weight-loss diets (Atkins, Protein Power Plan, Ornish, Weight Watchers and Zone) had sufficient evidence to suggest their use by people with diabetes whose lifestyles and personal preferences were congruent with the diets ${ }^{(7)}$. Other authors have considered that although some types of diets might be effective against obesity (low-calorie or protein/fat-rich diets), they have their limitations, such as the high dropout rates due to maintenance difficulties or the attenuation of only some, not all, of the components involved in this condition. Therefore, it is not yet known which dietary strategy is the most appropriate. A current consideration is the time of day that foods are consumed (food timing) ${ }^{(8)}$.

The fourth paragraph of the paper should begin:

On the other hand, recent studies conducted in humans suggest that factors related to the meal times, such as eating before or after a certain hour, restricting eating hours, allocating time for meals, the macronutrient composition of each meal of the day ${ }^{(8)}$, and even variety of the diet may also have an important role in total energy intake and therefore in the regulation of adiposity and body weight ${ }^{(12-14)} \ldots$ 